\title{
Screening of Tuberculosis Using Artificial Neural Network
}

\author{
A. Anand Kumar ${ }^{1 *}$, T. Mani², S. Gokulnath ${ }^{3}$, S. K. Kabilesh', K. Dinakaran ${ }^{5}$, A. Stephen Sagayaraj ${ }^{6}$ \\ ${ }_{1,2,3,4,5,6}$ Assistant Professor, Department of Electronics and Communication Engineering, Jai Shriram \\ Engineering College, Tirupur, India \\ *Corresponding author: anandkumar.a88@gmail.com
}

\begin{abstract}
Tuberculosis is an infectious bacterial disease that most commonly affects the lungs. This paper reviews, screening of tuberculosis in chest radiograph images using an artificial neural network (ANN). Implementing image processing techniques having segmentation, feature extraction from chest radiographs, at that point building up a fake neural organization for programmed characterization dependent on back proliferation calculation to group tuberculosis accurately. The performance was evaluated using SVM and ANN classifiers regarding exactness, review, and precision. The trial results Confirm the effectiveness of the proposed strategy that gives great Classification proficiency.
\end{abstract}

Keywords: Computer-helped location and finding, Division, Lung design acknowledgment and order, Tuberculosis (TB), Xbeam imaging.

\section{Introduction}

The strategy used to speak to of the inside of a body for clinical examination and clinical intercession is known as Medical image processing. Clinical imaging looks to uncover inward structures covered up by the skin and bones, just as to analyze and treat ailment [1]. In the Medical and healthcare sector, medical diagnosis is commonly handled with help of image processing and so Image-based clinical finding is one of the significant assistance zones. Medical images are plays an important role to get knowledge about the anatomical structures, diseases, and treatment planning [2], [16].

Tuberculosis, commonly known as TB, is a bacterial infection disease that is commonly found in the lungs. Tuberculosis is identified by finding Mycobacterium tuberculosis bacteria taken from the patient in a clinical specimen. A medical evaluation for tuberculosis (TB) includes a physical examination, a chest $\mathrm{X}$-ray, and microbiological examination. The chest $\mathrm{x}$-rays are important in helping to decide on the most effective treatment for TB. The chest X-rays are important in helping to decide on the most effective treatment for TB [15]. An accurate decision was taken by the physicians with help of computer-aided diagnosis to improve clinician's confidence in the analysis of medical images. Computer-based systems can design by utilizing counterfeit neural organization procedures. Counterfeit neural organization and fuzzy rationale are utilized for picture arrangement strategies in the territory of clinical determination [7].

Clinical science assists with forestalling and analyzes the infections by analyzing medical images. Artificial neural networks are applied in medical sciences for demonstrative frameworks, biomedical investigation, picture examination, and medication advancement. By utilizing counterfeit neural organizations, a ton of wellbeing boundaries (breath rate, circulatory strain, glucose level) are monitored. Artificial neural networks play important roles in image analysis with the processing of the digital image in recognition and classification. The medical image field is very important because it offers a lot of useful information for diagnosis and therapy [11]. There are four motivations to utilize ANN as a classifier: (I) furnish profoundly precise outcomes in correlation with relapse models (ii) Loads speaking to the arrangement are found by iterative preparing, (iii) Neural organizations models can be effectively refreshed. It implies they are reasonable for dynamic condition and (IV) speculation property of the ANN produces fitting outcomes for the information vectors that are absent in the preparation set. [12]. This paper discloses how to segregate among typical and unusual appearances of TB CXRs. A mechanized methodology for recognizing Tuberculosis appearances in chest X-beams (CXRs), in light of lung division and lung sickness characterization is created. A computerized way to deal with permits mass screening of enormous populaces that couldn't be overseen physically in X-beams. A banner foremost radiograph (X-beam) of a patient's chest is an obligatory aspect of each assessment for TB. A reliable screening system for TB detection using radiographs is more powerful in TB diagnostics. Lung division, include calculation, and grouping is the stepwise process that is used to a screening of TB in chest radiographs.

\section{Proposed Method}

The proposed strategy contains three phases: lung division, highlight extraction, and back-proliferation as a classifier. Figure 1 shows the outline of the proposed Back-Propagation Artificial Neural Network (BPANN) technique.

Fig. 1 shows the architecture of the system with the various processing steps are first, fragments the lung of the info CXR utilizing a chart cut enhancement strategy along with a 
lung model. For the portioned lung field, our framework at that point processes a gathering of highlights as a contribution to a pre-prepared parallel classifier. Utilizing choice calculations and edges, the classifier yields its trust in grouping the info CXR as a TB positive case.

The method to address the chest classification problem, presenting a generic approach for the diagnosis of chest pathologies of all types. The algorithm is comprised of two main phases: The image processing phase and the classification phase. The image processing phase includes the segmentation and feature extraction. The classification phase deals with the ANN classifier.

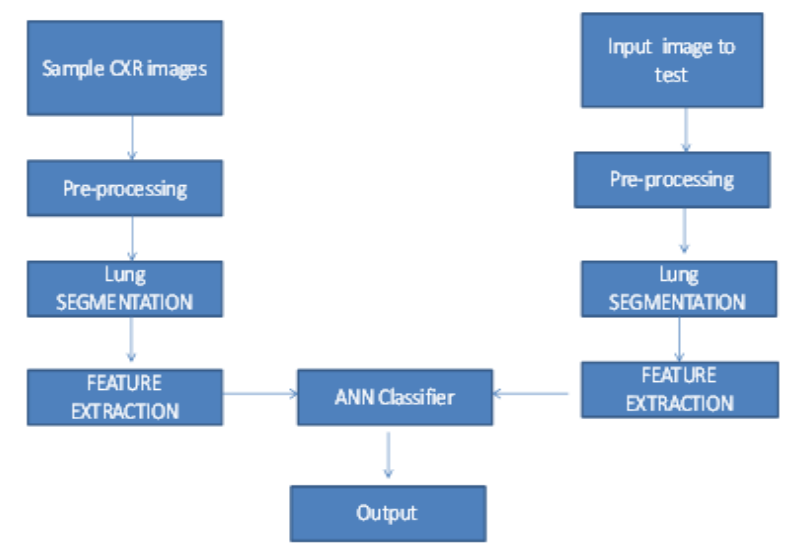

Fig. 1. System architecture

\section{A. Lung Segmentation}

Lung segmentation is performed by considering the properties of lung boundaries, regions, and shapes. The system has two principal stages. It first computes a mean shape model utilizing preparing pictures. At that point, it utilizes a chart slice division calculation to distinguish the lung Regions with the assistance of the determined shape model. In general, segmentation in clinical pictures has to cope with anatomical shape variations, poor contrast, and acquisition noise due to hardware constraints. Lung segmentation also faces these issues. Therefore, fuse a lung model that speaks to the regular lung state of chosen preparing veils. Select these veils as per their shape comparability as follows. All preparation covers are directly adjusted to a given info CXR. At that point process, the vertical and flat force projections of the histogram balanced pictures. To quantify the similarity between projections of the information CXR and the preparation CXRs, utilize the Bhattacharyya coefficient.

To compute an approximate shape model, use training images which are selected according to their shape similarity. The average of all selected masks is employed as an approximate shape model for the observed patient lung image. The next stage of the system identifies the lung region with a segmentation algorithm.
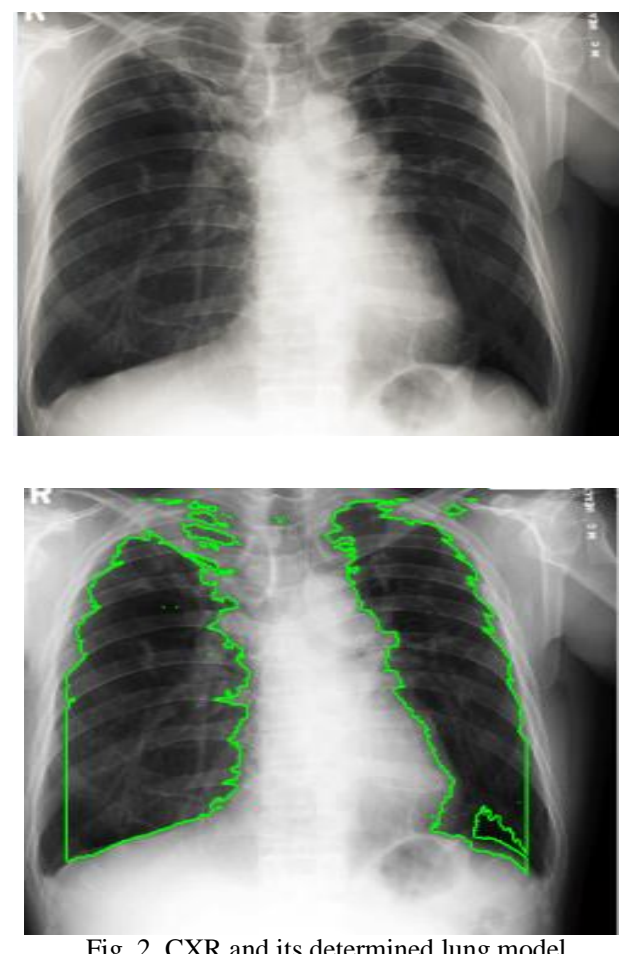

Fig. 2 shows a CXR and its determined lung model. Chart cut methodology and models the lung limit discovery with a goal work. To detail the objective capacity, characterize three prerequisites a lung locale must fulfill: I) The lung area ought to be as per run of the mill CXR forces expected during a lung district, ii) Neighboring pixels ought to have steady names, iii) The lung area should be like the lung model figured [17].

\section{B. Features}

To describe normal and abnormal patterns in the segmented lung field, experimented with two different feature sets. Our motivation is to use features that can pick up subtle structures in a CXR.

\section{1) CXR and its determined lung model}

Fig. 2 shows a CXR and its determined lung model. Chart cut methodology and models the lung limit discovery with a goal work. To figure the objective capacity, characterize three prerequisites a lung district must fulfill:

a) The lung area ought to be as indicated by regular CXR powers expected during a lung locale,

b) Neighbouring pixels ought to have steady marks,

c) The lung area should be like the lung model registered.

The primary set incorporates shape, edge, and surface descriptors. For every descriptor, register a histogram that shows the conveyance of the distinctive descriptor esteems over the lung field. Every histogram canister is a component, and all highlights of all descriptors set up structure an element vector that contribution to our classifier. Through observational analyses, discovered that utilizing 32 containers for every histogram gives us great down to earth results. Specifically, 
utilize the accompanying shape and surface descriptors.
a) Intensity histograms $(\mathrm{IH})$
b) Gradient Magnitude Histograms (GM)
c) Shape descriptor histograms (SD)
d) $\mathrm{SD}=\tan -1(\lambda 1 / \lambda 2)$

$\lambda 1, \lambda 2$-the Eigen estimations of a Hessian grid with $\lambda 1 \leq \lambda 2$.

Histogram of situated slopes (HOG) might be a descriptor for inclination directions weighted predictable with angle size. The picture is part of little associated districts, and for each area, a histogram of slope course or edge directions for pixels inside the locale is registered. The mix of these histograms speaks to the descriptor.

2) CBIR-based Image Features - Set B

The component assortments incorporate force, edge, surface, and shape second highlights, which are normally utilized by CBIR frameworks. Inspect the impact of high dimensional element spaces on order exactness. Concentrate a large number of the highlights, aside from the $\mathrm{Hu}$ minutes and shape highlights.

Tamura surface descriptor: The Tamura descriptor is inspired by human viewing. The descriptor includes a lot of six highlights. Just utilize three of those highlights, which have the most grounded connection with human recognition: differentiation, directionality, and coarseness.

\section{Classification}

One of the most widely recognized ANN utilized for groupings is the feed-forward organization. In a feed-forward organization, the neurons in each layer are just associated with the neurons in the following layer. Feed-forward organizations regularly utilize the back-proliferation (B-P) administered learning calculation to powerfully change the weight and predisposition esteems for every neuron in the organization.

Back proliferation neural organizations utilize one of the most famous neural organization learning calculations, the Back engendering (BP) calculation. The back engendering calculation prepares a given feed-forward multi-layer neural organization for a given arrangement of information designs with known groupings. At the point when every passage of the example set is given to the organization, the organization assesses its yield reaction to the example input design. The yield reaction is contrasted with the known incentive with expected yield and the mistake esteem is determined. Because of the mistake, the association loads are balanced. The back engendering calculation depends on the Windrow-Hoff delta learning rule in which the weight modification is done through the mean square blunder of the yield reaction to the example.

\section{Preparation stage comprises of five significant advances:}

- Load a picture from an information base.

- As discussed earlier extricate the highlights from the selected features of the trained images after the image preprocessing method.

- Extracted features (feature vector) are applied as input to BP Algorithm.
- Training the backpropagation neural network.

- Save as .mat file.

\section{E. Testing stage consists of five major steps}

- Loading of an image to be tested from a database.

- Apply image pre-processing and features extraction techniques to the input image application of extracted features as input to a trained Neural network.

- Dynamic by B-P calculation as per the estimation of highlights.

- Checking yield created from a neural organization

\section{Results}

The example pictures that have been taken from the accessible assets are sifted utilizing the Median Filtering procedure. The Median separating strategy is utilized to eliminate the clamors which the client can't relate to the unaided eye. The Median channel in this manner improves the nature of the pictures. The example pictures are given to the process of lung segmentation followed by the appropriate filter method. The lung division measure just fragments the lung locale from the example of $x$-beam pictures. The example pictures after applied the division method are given to the Feature extraction. The overall process can be shown by using the GUI window.

The input images of Chest X-Rays fed to the system and it results in certainty esteem that shows the level of an anomaly for the information pictures. To assess the exhibition of this work, Sensitivity, Specificity, and Accuracy was utilized and the measures are resisted as follows.

$$
\begin{gathered}
\text { Accuracy }=\frac{\text { Total number of testing samples }}{\text { Number of correctly classified testing samples }} \\
\text { Sensitivity }=\frac{\text { True positives }}{\text { True positives }+ \text { False positives }} \\
\text { Specificity }=\frac{\text { True positives }}{\text { Genuine positives }+ \text { False positives }}
\end{gathered}
$$

Where True positives mean accurately recognized, bogus positives indicate erroneously distinguished, bogus negative signifies mistakenly denied.

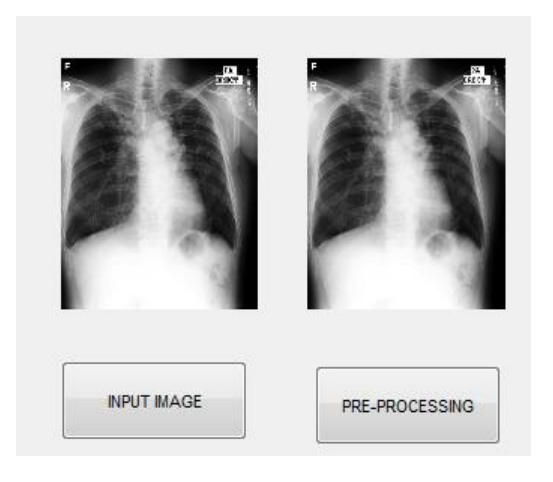



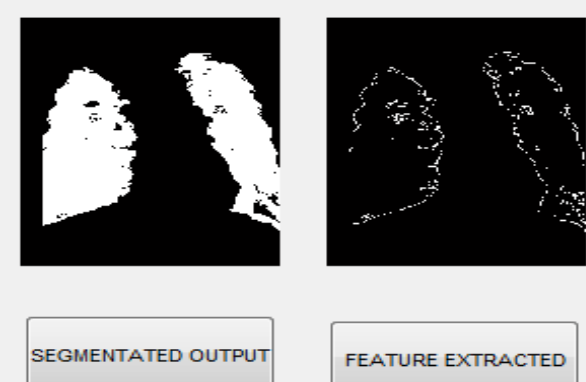

FEATURE EXTRACTED

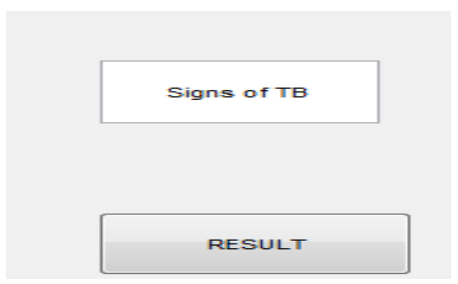

Fig. 3. System overview

Table 1

Comparison between SVM and ANN classifier

\begin{tabular}{|c|c|c|}
\hline Technique Used & ANN & SVM \\
\hline No. of images & 11 & 11 \\
\hline Accuracy & $93 \%$ & $85 \%$ \\
\hline Sensitivity & 83 & 76 \\
\hline Specificity & 50 & 54 \\
\hline
\end{tabular}

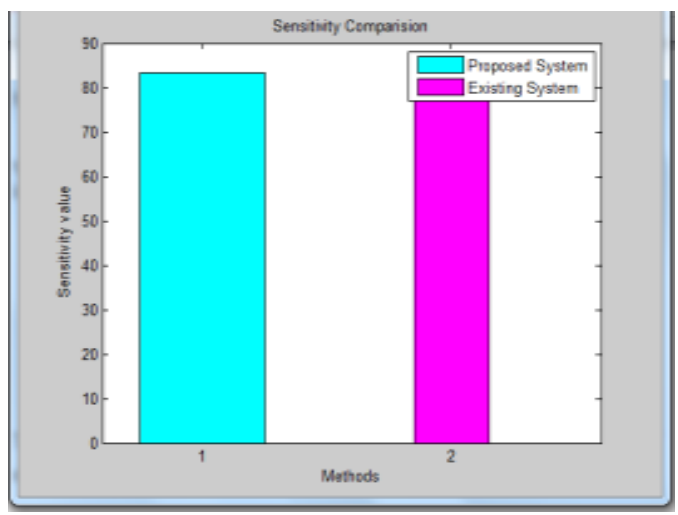

(a) Sensitivity

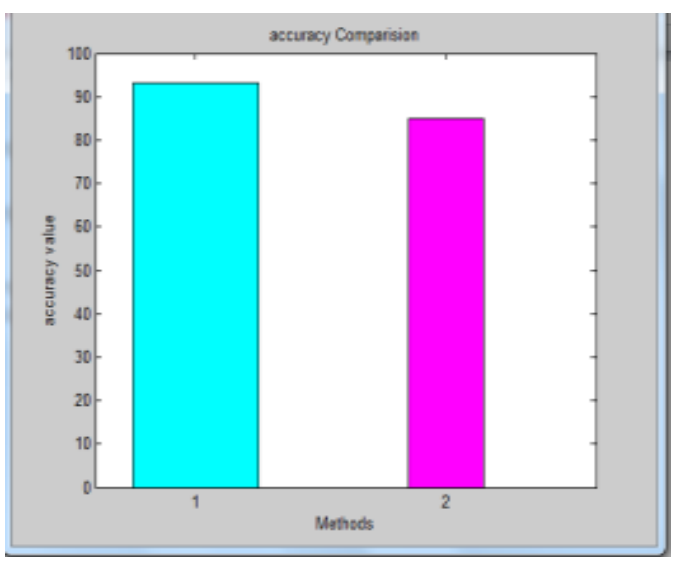

(b) Accuracy

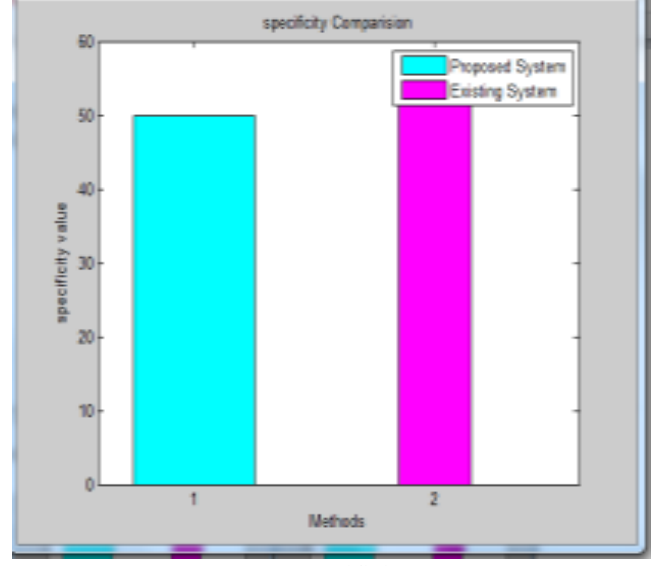

(c) Specificity

Fig. 4. Comparisons of SVM and ANN

Table 1, shows the comparison of SVM with the ANN classifier. It can be seen that by using the ANN classifier it is possible to achieve $93 \%$ accuracy compared to the previous method that is the SVM classifier. The sensitivity rate is also high in the ANN classifier compared to the SVM. The comparison analysis between the SVM and ANN classifier, in terms of sensitivity, specificity, accuracy is graphically shown in figure 4.

\section{Conclusion}

A mechanized framework that screens CXRs for signs of TB has been created dependent on picture handling and neural organization innovation utilizing feed-forward neural organization prepared by the back-spread calculation. The lung region is extracted using a graph cut segmentation method. A set of texture and shape features are computed for the segmented lung region, which is given as a contribution to the Input to the B-P ANN which is used to distinguish Tuberculosis in CXR pictures. The exhibitions of the arrangement calculation are assessed utilizing exactness rate and it compared with existing SVM classification. From the result analysis, the ANN classifier has more accuracy compared to the SVM classifier. The performance of the system is analyzed using a MATLAB simulator.

\section{References}

[1] Stefan Jaeger," Automatic Tuberculosis Screening Using Chest Radiographs", IEEE transactions on medical imaging, vol. 33, no. 2, February 2014.

[2] S. Candemir, S. Jaeger, K. Palaniappan, S. Antani, and G. Thoma, "Graph-cut based automatic lung boundary detection in chest radiographs," in Proc. IEEE Healthcare Technol. Conf.: Translate. Eng. Health Med., 2012, pp. 31-34.

[3] S. Candemir, K. Palaniappan, and Y. Akgul, "Multi-class regularization parameter learning for graph cut image segmentation," in Proc. Int. Symp. Biomed. Imag., 2013, pp. 1473-1476.

[4] S. Jaeger, A. Karargyris, S. Antani, and G. Thoma, "Detecting tuberculosis in radiographs using combined lung masks," in Proc. Int. Conf. IEEE Eng. Med. Biol. Soc., 2012, pp. 4978-4981

[5] C. Leung, "Reexamining the role of radiography in tuberculosis case finding," Int. J. Tuberculosis Lung Disease, vol. 15, no. 10, pp. 12791279,2011 
[6] Yoshihiko Nakamura, Gentaro Fukano, Hotaka Takizawa, Shinji Mizuno, Shinji Yamamo, Tohru Matsumoto, Yukio Tateno, and Takeshi Iinuma. "Eigen Nodule: View-based Recognition of Lung Nodule in Chest Xray CT Images Using Subspace Method," International Conference on Pattern Recognition, vol. 4, pp. 681-684, 2004.

[7] J. Wei, Y. Hagihara, A. Shimizu, and H. Kobatake. "Optima limage feature set for detecting lung nodules on chest x-ray images," in Proc. Int. Workshop on Computer-Aided Diagnosis, Citeseer, 2002.

[8] Yoshida. "Local contralateral subtraction based on bilateral symmetry of lung for reduction of false positives in computerized detection of pulmonary nodules," IEEE Transactions on Biomedical Engineering, 2004.

[9] UriAvni, Hayit Greenspan, EliKonen, Michal Sharon, and Jacob Goldbergeri. "X-ray Categorization and Retrieval on the Organ and Pathology Level, Using Patch-Based Visual Words," IEEE Transactions on Medical Imaging, vol. 30, 2011.

[10] S. Jaeger, A. Karargyris, S. Candemir, J. Siegelman, L. Folio, S.Antani, and G. Thoma, "Automatic screening for tuberculosis in chest radiographs: A survey," Quant. Imag. Med. Surg, 2013.

[11] B. Van Ginneken, B. ter Haar Romeny, and M. Viergever, "Computeraided diagnosis in chest radiography: A survey," IEEE Trans. Med. Imag., Dec. 2001.
[12] S. Kakeda, J. Moriya, H. Sato, T. Aoki, H. Watanabe, H. Nakata, N. Oda, S. Katsuragawa, K. Yamamoto, and K. Doi, "Improved detection of lung nodules on chest radiographs using a commercial computer-aided diagnosis system," Am. J. Roentgenol., 2004.

[13] A. Dawoud, "Fusing shape information in lung segmentation in chest radiographs," Image Anal. Recognit., 2010.

[14] P. Maduskar, L. Hogeweg, H. Ayles, and B. van Ginneken, "Performance Evaluation of automatic chest radiograph reading for detection of tuberculosis (TB): A comparative study with clinical officers and certified readers on TB suspects in sub-Saharan Africa," in Eur. Congr. Radiol., 2013.

[15] Díaz-Huerta JL, Téllez-Anguiano AdC, Fraga-Aguilar M, GutiérrezGnecchi JA, Arellano-Calderón S "Image processing for AFB segmentation in bacilloscopies of pulmonary tuberculosis diagnosis" PLoS ONE, vol. 14, no. 7, 2019.

[16] Kabilesh, S. K., et al. "Medical Image Counterfeit Detection for Smart Healthcare," Journal of Sensor Research and Technologies, 2020.

[17] Sagayaraj, A. Stephen, et al. "Segmentation of Breast Cancer Using Texture Method in Mammogram Image," in International Journal of Modern Trends in Engineering and Science, vol. 4, no. 3, pp. 193-195, 2017. 